\title{
An adaptive energy-aware transmission scheme for wireless sensor networks
}

\author{
Abdullahi Ibrahim Abdu, Muhammed Salamah \\ Computer Engineering Department, Eastern Mediterranean University, KKTC, Mersin 10, TURKEY
}

Email address:

teetex4sure@gmail.com (A. I. Abdu), muhammed.salamah@emu.edu.tr (M. Salamah)

\section{To cite this article}

Abdullahi Ibrahim Abdu, Muhammed Salamah. An Adaptive Energy-Aware Transmission Scheme for Wireless Sensor Networks, International Journal of Wireless Communications and Mobile Computing. Vol. 1, No. 1, 2013, pp.14-20. doi: 10.11648/j.wcmc.20130101.13

\begin{abstract}
In this paper, we proposed a scheme to extend the lifetime of a wireless sensor network, in which each sensor node decides whether to transmit a message or not and with what range to transmit the message, based on its own energy reserve level and the information contained in each message. The information content in each message is determined through a system of rules describing prospective events in the sensed environment, and how important such events are. The messages deemed to be important are propagated by all sensor nodes and with different transmission ranges depending on nodes energy resource level, while messages deemed to be less important are handled by only the nodes with high energy reserves level and transmitted with different transmission ranges based on nodes energy resource level. The results show that by adapting the transmission range based on nodes energy reserve and message importance, a considerable increase in network lifetime and connectivity can be achieved.
\end{abstract}

Keywords: Energy-Aware, Wireless Sensor Networks, Adaptive Transmission Ranges, Priority Balancing

\section{Introduction}

A wireless sensor network (WSN) typically consists of a number of small, inexpensive, locally powered sensor nodes that communicate detected events wirelessly through multi-hop routing [1]. These networks have limited computing capability and memory [2]. Typically, a sensor node is a tiny device that includes three basic components: a sensing subsystem for data acquisition from the physical surrounding environment, a processing subsystem for local data processing and storage, and a wireless communication subsystem for data transmission. In addition, a power source supplies the energy needed by the device to perform the programmed task. This power source often consists of a battery with a limited energy budget. In addition, it could be impossible or inconvenient to recharge the battery, because nodes may be deployed in a hostile or unpractical environment [3]. WSNs are being used in a wide variety of critical applications such as traffic, defense, medical treatment, manufacturing [4], military, health-care applications [5-6], and environmental monitoring [7]. A key research area is concerned with overcoming the limited network lifetime inherent in the small, locally powered sensor nodes [1]. Many of the WSN algorithms designed to extend the network lifetime are modified routing algorithms [8]. How- ever, a low cost and low complexity technique is the motivation of this study.

In this paper, a scheme to extend the lifetime of a wireless sensor network, named as AIRT (Adaptive Information managed energy aware algorithm for sensor networks with Rule managed reporting and Transmission range adjustments) is proposed. The extension in the network lifetime is achieved by adapting transmission ranges based on nodes energy reserve level and importance of messages. One main advantage of this scheme is that, nodes do not have to transmit messages with their maximum transmission ranges all the time. The node's energy reserve level and message importance are taken into consideration, and therefore the node's transmission range is adapted accordingly. AIRT also maintains high network connectivity by adjusting the minimum transmission range of a node to cover at least one other sensor node.

The rest of this paper is organized as follows: Section 2 presents the related work. Section 3 presents the proposed AIRT scheme. Section 4 gives the performance study (simulation results and discussions). Section 5 provides conclusion and future work.

\section{Related Work}

In Merret et. al. [9], IDEALS (Information manageD 
Energy aware ALgorithm for Sensor networks) was introduced as a scheme that extends the lifetime of a WSN through the possible discrimination of low important messages and prioritizing important messages. The IDEALS scheme is built upon the idea of message priorities and power priorities.

Authors in [10] proposed a localized technique to extend the lifetime of a wireless sensor network; referred to as IDEALS|RMR (Information manageD Energy aware ALgorithm for Sensor networks with Rule Managed Reporting). The extension in the network lifetime is achieved at the possible sacrifice of low importance packets, as a result of a union between: Information control, quantified by a system of rules, referred to as rule managed reporting (RMR) and energy management, supplemented by energy harvesting, for example, mechanical vibrations. Furthermore, IDEALS|RMR uses a single-fixed transmission range for each sensor node regardless of whether its energy resource is high or low and this causes redundancy in energy consumption as lots of areas are covered by several sensors.

In Mingming et al. [11], the authors developed a power saving technique by combining two methods: scheduling sensors to alternate between active and sleep mode method, and adjusting sensors sensing range method. They combined both methods by dynamic management of node duty cycles in a high target density environment. In their approach, any sensor schedules its sensing ranges from 0 to its maximum range, where 0 corresponds to sleep mode.Adding the system of information control proposed in this paper could significantly save energy.

Authors in [12] try to deal with the problem of energy holes (unbalance distribution of communication loads) in a wireless sensor network. This means that, energy of nodes in a hole will be exhausted sooner than nodes in other region. As energy holes are the key factors that affects the life time of wireless sensor network, they proposed an improved corona model with levels for analyzing sensors with adjustable transmission ranges in a WSN with circular multi-hop deployment. Additionally, the authors proposed two algorithms for assigning the transmission ranges of sensors in each corona for different node distributions. These two algorithms reduce the searching complexity as well as provide results approximated as optimal solution.

Two forwarding techniques were proposed by M. Busse et al. in [13] to maximize energy efficiency. The techniques are termed single-link and multi-link energy efficient forwarding. Single-link forwarding sends a packet to only one forwarding node; multi-link forwarding exploits the broadcast characteristics of the wireless medium. This leads to efficiency because if one node does not receive a packet, another node will receive the packet and performs the forwarding process. There is however a tradeoff of delivery ratios against energy costs.

In [14], we extended the lifetime of wireless sensor network by sacrificing low importance message and also adjusting of transmission ranges based on only nodes energy reserve. However, in our proposed AIRT technique, we improved lifetime even better by adapting transmission ranges based not only on nodes energy reserve, but also on message importance.

\section{The Proposed AIRT Scheme}

The idea of our proposed AIRT scheme was first derived from [9], where the basic principles of IDEALS (Information manageD Energy aware ALgorithm for Sensor networks) was introduced as a scheme providing an increase in the lifetime of a wireless sensor network through the discrimination of certain messages. Additionally, the work of [9] was extended by authors in [10] through coupling IDEALS with a system for determining the messages information content - RMR (Rule Managed Reporting). Furthermore, in our previous research (named as IRT) [14], we extended [10] by introducing adjustable transmission ranges based on nodes energy reserve only. Finally, in this paper, we extended our previous work [14] by

- Coupling IDEALS|RMR with transmission range adjustment (TRA), whereby the TRA is determined as a function of two parameters;

1. Nodes energy reserve level and

2. Importance of the message.

- Perform a detailed analysis through the simulation of the AIRT, IRT [14], IDEALS|RMR [9, 10], and traditional $[9,10]$ schemes to prove that the proposed AIRT technique is the most energy efficient.

The system diagram of the proposed AIRT scheme is shown in Fig. 1. Firstly, upon sensing data, the sensor node passes the information to the controller, which sends the sensed value (e.g. temperature) to RMR (Rule Management Reporting) unit. The purpose of RMR unit is to determine if a message worth reporting has occurred and how important that message is. The value is then received by the Rule Testing module. Its responsibility is to determine if a message worth reporting has occurred. It does that by checking the sensed data against the rules in the Rule Database module (getting history information about the previously sensed values), at the same time, it updates the history with the current information of message and sensed message. Rules may be fulfilled or not, any rules which are fulfilled are passed to the Message Priority Assignment Module to determine how important the content of the message is. It does that by assigning message priorities (MP) to each fulfilled rule. In this work, five different MPs are used (MP1MP5). MP1 denotes the most important message which might represent drastic change in the sensed data value. Conversely, MP4 - MP5 relates to the least important messages which might represents slight or no change in the sensed value. MP2 - MP3 relates to intermediate priorities messages which might represent moderate change in the sensed value. Any number of predefined rules can be entered by the designer, and these rules describe different events that can be detected in the sensed environment. Examples of such possible rules are: 


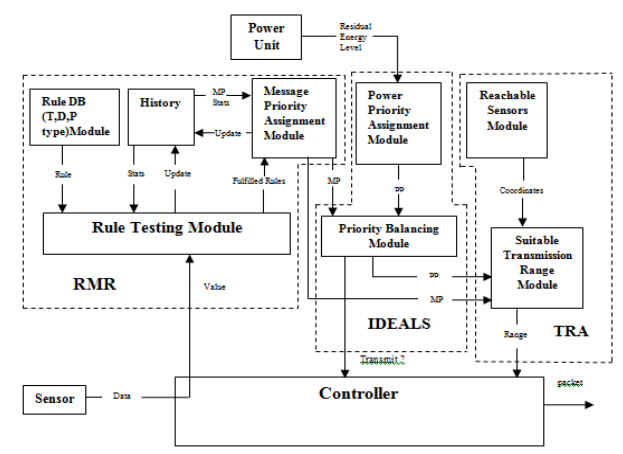

Fig. 1. The proposed AIRT system diagram.

1. Threshold rules (applied when the sensed value crosses a preset value).

2. Differential rules (applied when the change in the sensed value is larger or smaller than a preset value).

3. Routine rules (applied when a message of a certain importance has not been sent for a preset period) [10].

Secondly, data from RMR is then passed to IDEALS unit, its responsibility is to decide if the node should transmit a message or not, and it's done by Priority Balancing Module. The node's energy resource is characterized by Power Priority Assignment Module, which assigns a power priority (PP) for each node based on the state of its battery. A full battery life is assumed to have 100jouls of energy, whereas an empty battery life is assumed to be 0jouls. The 100jouls is divided equally into 5 levels, and priorities are assigned to each level. The highest power priority is PP5 and it is assigned to the highest level of energy for a node which is from 80 to $100 \mathrm{j}$. While the lowest power priority is PP1 and it is assigned to the lowest level of energy for a node which is from 0 to $20 \mathrm{j}$. When priority balancing module receives MP and PP, it compares them and if PP $\geq$ MP, then the message will be transmitted as illustrated in the priority allocation and balancing process of Figure 2.

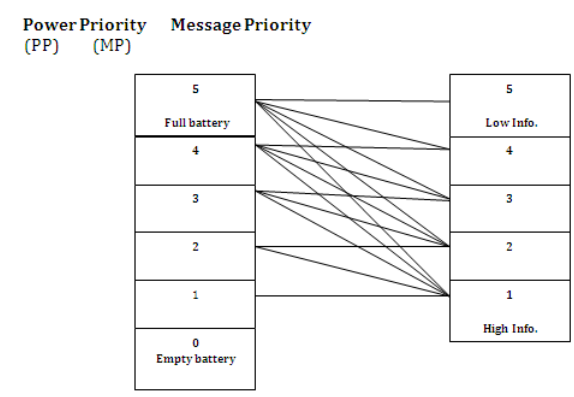

Fig. 2. Priority Balancing

Finally, PP and MP are also passed to the Transmission Range Adjustment (TRA) unit in which the Suitable Transmission Range module decides with what range a sensor node shall transmit the message. The Suitable transmission range module gets PP from the power priority allocation module, MP from message priority allocation module and coordinates from reachable sensors module. The reachable sensors are the entire sensors in the maximum transmission range of a sending sensor node. Now, based on the value of the PP, and MP, a suitable transmission range (TR) is determined and passed to the controller to transmit the message with the new range as illustrated in the heuristic Table 1. In this work, five different TRs are used (TR1-TR5), where TR1 represents minimum transmission range and TR5 represents maximum transmission range. For example, when a node has full battery (i.e. PP5), it will transmit MP1 messages with TR5, MP2 with TR4, MP3 with TR3, MP4 with TR2 and MP5 with TR1. However, if a node's battery decreases to its minimum PP1, it will transmit only messages with the highest message priority MP1 and with the lowest transmission range TR1. It is worth to mention that a power control mechanism is assumed to be embedded in the sensor node for the adjustment of its transmission range.

Table 1. Heuristically determined transmission ranges.

\begin{tabular}{llllll}
\hline $\begin{array}{l}\text { MP } \\
\mathbf{P P}\end{array}$ & $\mathbf{5}$ (Highest) & $\mathbf{4}$ & $\mathbf{3}$ & $\mathbf{2}$ & $\mathbf{1}$ (Lowest) \\
\hline 5 (Lowest) & 1 (Min. TR) & 0 & 0 & 0 & 0 \\
4 & 2 & 2 & 0 & 0 & 0 \\
3 & 3 & 4 & 2 & 0 & 0 \\
2 & 4 & 4 & 3 & 2 & 0 \\
1 (Highest) & 5 (Max. TR) & 4 & 3 & 2 & 2 \\
\hline
\end{tabular}

The transmission ranges in Table 1 above were determined heuristically after several runs of simulation with different values of TR. The resultant matrix has a meaningful structure which reflects the tradeoffs between MP and PP. These near-optimal TR values for the AIRT scheme improve the network lifetime, connectivity, and maintain a considerable message loss compared to other schemes (IRT, IDEALS|RMR, and Traditional) as will be seen in the performance analysis section.

Table 1 can be seen as a matrix A with $i=\mathrm{MP}, j=\mathrm{PP}$, and $\mathrm{A}[i, j]=\mathrm{TR}$. Then, assuming that the number of levels used for MP, PP and TR is equal to $\mathrm{L}$, the transmission ranges can be derived from the following algorithm:

$$
A[i, j]=\left\{\begin{array}{cl}
0 & \text { if } i>j \\
2 & \text { if } i=j \text { and } j \neq L \\
j & \text { if } i<j \text { and } j \neq L \\
L+1-j & \text { if } j=L
\end{array}\right.
$$

\section{Performance Evaluation}

Performance evaluation of the proposed AIRT scheme is done through extensive simulation. To show that the proposed scheme conserves the most amount of energy, it is compared with other related power saving schemes based on Energy Depletion Times (EDT) and Network Connectivity (NC) performance metrics. Based on these perfor- 
mance metrics, the simulations of Traditional, IDEALS|RMR, IRT, and AIRT schemes are conducted.

\subsection{Simulation Setup}

We simulated 20 sensor nodes in a network size of $70 \times 70$ meters. Each node has the same maximum transmission range of 20 meters. A predefined node placement topology has been considered in this study as it can be seen from Figure 4. At the beginning of the simulation, all nodes have the same initial energy of 100 Joules [10]. The following Equation [10] is used for calculating the energy required to transmit a packet:

$$
E_{\mathrm{tx}}(1, d)=E_{\mathrm{elec}} l+E_{\mathrm{amp}} l d^{2}
$$

where $E_{\text {elec }}[\mathrm{J}]$ is the energy required for the circuitry to transmit or receive a single bit, $E_{\text {amp }}[\mathrm{J}]$ is the energy required for the transmit amplifier to transmit a single bit a distance of one meter, $d$ is the separation distance in meters and $l$ is the length of the packet. The simulation parameters are given in Table 2 .

Table 2. Simulation parameters and results.

\begin{tabular}{|c|c|}
\hline Simulation area & $70 \times 70$ meters \\
\hline Number of nodes & 20 nodes \\
\hline Packet length & 1000 bytes \\
\hline Initial node energy & 100 Joules \\
\hline Simulated Node Id & node- 08 \\
\hline Minimum transmission range & 13.04 meters \\
\hline Maximum transmission range & 20 meters \\
\hline Simulated node Coordinates & $(x=38, y=37)$ \\
\hline Waiting time ' $\mathrm{t}$ ' & $\mathrm{t}$ minutes \\
\hline $\begin{array}{l}\text { Node } 08 \text { Traditional Scheme } \\
\text { Result, for NEDT performance } \\
\text { Metric. }\end{array}$ & 9 Hours \\
\hline $\begin{array}{l}\text { Node } 08 \text { IDEALS/RMR } \\
\text { Scheme Result, for NEDT } \\
\text { Performance Metric. }\end{array}$ & 34 Hours \\
\hline $\begin{array}{l}\text { Node } 08 \text { IRT Scheme } \\
\text { Result, for NEDT } \\
\text { Performance Metric. }\end{array}$ & 63 Hours \\
\hline $\begin{array}{l}\text { Node } 08 \text { AIRT Scheme } \\
\text { Result, forNEDT } \\
\text { Performance Metric. }\end{array}$ & 82 Hours \\
\hline $\begin{array}{l}\text { Node } 08 \text { Traditional } \\
\text { Scheme Result, for NC } \\
\text { Performance Metric. }\end{array}$ & 9 Hours \\
\hline $\begin{array}{l}\text { Node } 08 \text { IDEALS/RMR } \\
\text { Scheme Result, for NC } \\
\text { Performance Metric. }\end{array}$ & $\begin{array}{l}\text { MP5 MP4 MP3 MP2 MP1 } \\
4 \mathrm{~h} 11 \mathrm{~h} 12 \mathrm{~h} 22 \mathrm{~h} 34 \mathrm{~h}\end{array}$ \\
\hline
\end{tabular}

Node 08 IRT Scheme

Result, for NC

Performance Metric.

Node 08 AIRT Scheme

Result, for NC Performance Metric. 23h 53h57h68h82h

Only one sensor node is chosen for the simulation as the remaining sensors are assumed to operate in the same way. The chosen sensor node id and the distance to its closest sensor node are given as inputs. The sensor node senses data and the AIRT algorithm is performed as illustrated in Figure 3. Since the maximum transmission range (TR5) is fixed for every sensor, the other transmission ranges can be calculated by considering the minimum transmission range (TR1) as the distance to the closest sensor in the sending sensor's maximum transmission range. So the ranges between TR1 to TR5 are calculated successively by adding $\Delta \mathrm{TR}=(\mathrm{TR} 5-\mathrm{TR} 1) / 4$. That is, $\mathrm{TR}(\mathrm{i})=\mathrm{TR}(\mathrm{i}-1)+\Delta \mathrm{TR}$, for $\mathrm{i}=2,3,4$. For example, adding $\Delta \mathrm{TR}$ to TR1 gives TR2, and so on. To maintain connectivity, we took TR1 as the distance to the closest sensor in the sending sensor's transmission.

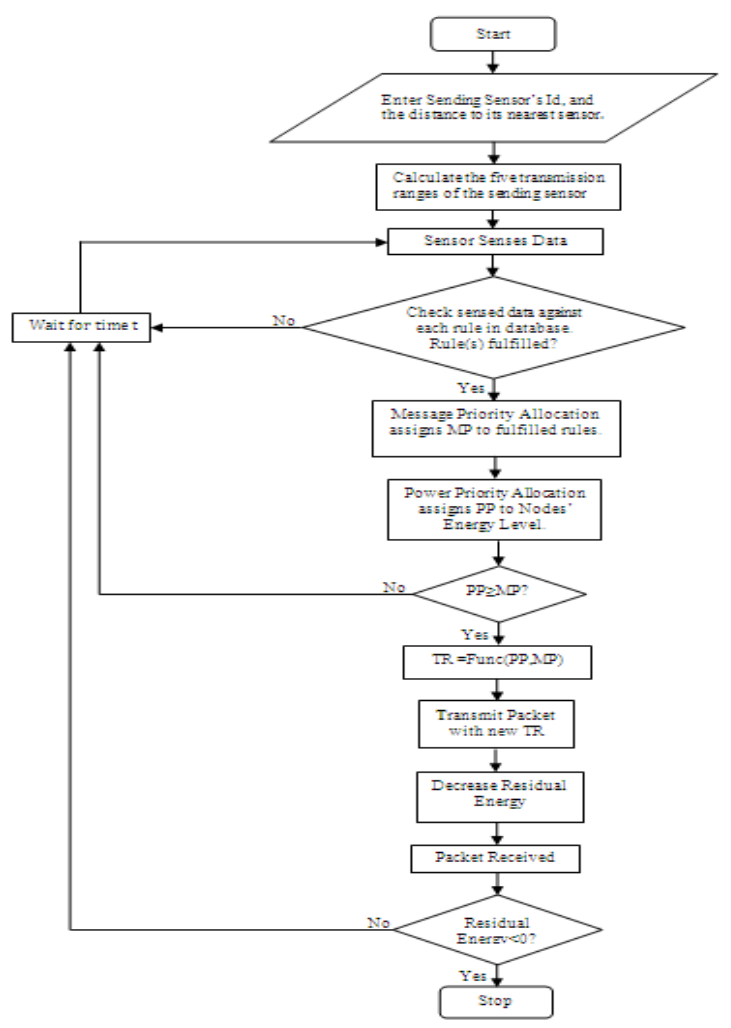

Fig. 3. Simulation Flowchart of AIRT scheme.

All nodes except the sink node (final destination of messages), performs multi-hop routing of messages by using the flooding algorithm. The simulation program is dynamic in the sense that, different coordinates from the ones used in the simulation can be entered and any node can be chosen for the simulation. Figure 4 shows a snap shot of a predefined node placement topology used in the simulation. Circles represent the maximum transmission range of sen- 
sors and lines represent possible communication link [10]. We chose node- 8 as it is located in the middle. We assumed messages are transmitted every 5 minutes.

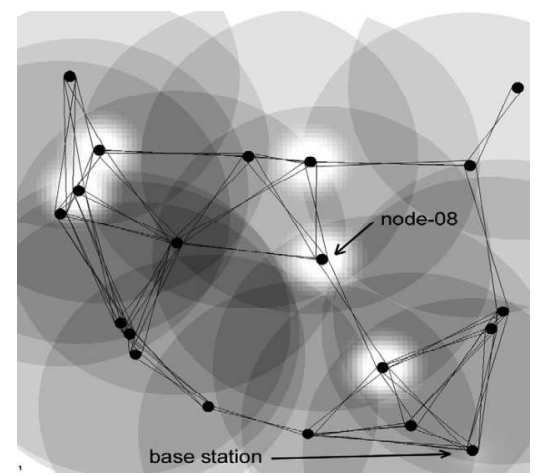

Fig. 4. A snap shot of node placement used in the simulation.

\subsection{Simulation Results}

Extensive simulation was done to show the effectiveness of the proposed AIRT scheme in extending the network life time. Rather than message generation, the simulator focused on the operation of our algorithm, whereby a data is sensed, if it is worth reporting based on user defined rules, priority is given to the related message, and the message is transmitted if $\mathrm{PP} \geq \mathrm{MP}$. Furthermore, based on data priority (MP) and battery life (PP), a suitable transmission range is determined using Table 1.

(1)Node Energy Depletion Times (NEDT): Fig. 5 shows node- 8 energy depletion times for all the four schemes. In the figure, ' 100 ' means that the node energy reserve is full, while ' 0 ' means that the energy reserve is depleted. For the traditional scheme, node- 8 depleted its energy reserve after around $9 \mathrm{~h}$, this is because it is transmitting messages regularly every 5 minutes, and does not take into account the nodes energy reserve and message importance. For the IDEALS|RMR scheme, it can be seen that the nodes energy level drops suddenly, this is because when the node has high energy reserve, it transmits every message that comes to it. The energy level then remains constant for a while because of less importance messages which are not transmitted. For the IRT scheme, the energy level drops and then remains constant because the IDEALS|RMR scheme is embedded inside IRT. However, the network lifetime increased almost twice that of the IDEALS|RMR due to the adjustment of transmission ranges based on the nodes energy reserve level. Finally, for the AIRT scheme, the same process of sudden dropping of energy level and then remaining constant occurs. However, the network lifetime is significantly enhanced compared to all other three schemes. The achieved improvements of the AIRT scheme over traditional, IDEALS|RMR, and IRT schemes are $720 \%, 135 \%$, and $33 \%$ respectively. This is due to the fact that nodes are adapting their transmission ranges based on both their energy reserve level and message importance.

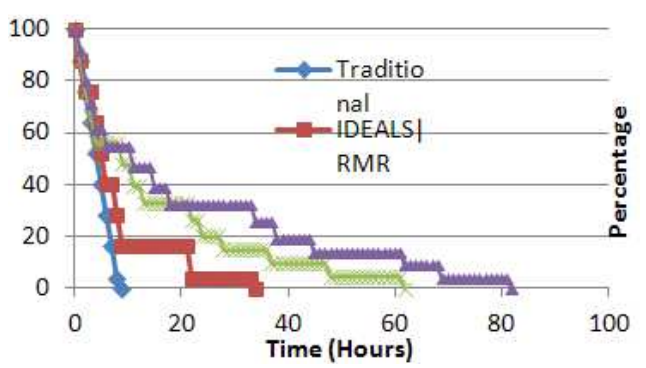

Fig. 5. Node energy depletion times.

(2) Network Connectivity (NC): The network connectivity is the measure of the ability of any node in the network to successfully transmit a message to the sink node. If the network is $100 \%$ connected, then any node in the network can successfully transmit a message to the sink node. However if the network is $50 \%$ connected, then only half of the nodes can successfully transmit a message to the sink node. Figure 6 shows that the connectivity of the Traditional scheme is completely lost after around $9 \mathrm{hrs}$. Figure 7 shows the connectivity of the IDEALS|RMR scheme. It is clear from the figure that this scheme extends the network lifetime and hence improves the network connectivity. The network lifetime for the most trivial message (MP5) is lost after around 4hrs (therefore no message of MP5 importance can reach the sink node after $4 \mathrm{hrs}$ ) while it is connected for about $76 \%$. However the network is still around $80 \%$ connected for the most important message (MP1) for $34 \mathrm{hrs}$. This is because the messages can not be transmitted every 5 minutes as in the traditional scheme. Figure 8 shows the performance of the IRT scheme. As it can be seen from the figure, IRT manages to extend the network lifetime almost twice that of the IDEALS/RMR scheme. This is because of the adjustment of transmission range based on nodes energy level. Additionally, network lifetime for the most trivial message is lost after around $13 \mathrm{hrs}$ while it is connected for about $76 \%$. However the network is $79.6 \%$ connected for the most important message for $63 \mathrm{hrs}$. Finally, Figure 9 shows the connectivity of the proposed AIRT scheme. It is obvious that AIRT scheme outperforms all other schemes as it has the longest network lifetime and maximum connectivity. It is interesting to note that AIRT extends the network lifetime twice longer that of IDEALS/RMR scheme and a little longer than the IRT scheme for MP5. Despite its lifetime finishes in 83 hours, the network is $84.7 \%$ connected for the most important message MP1.

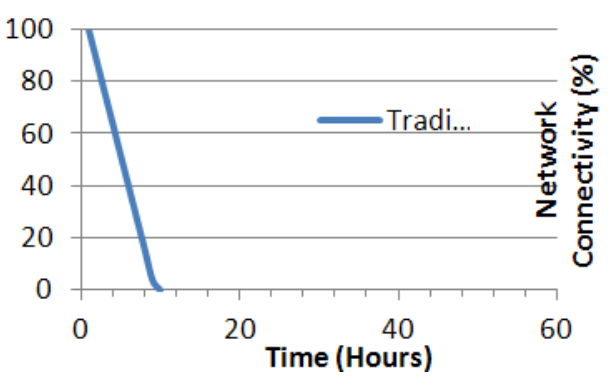

Fig. 6. Connectivity of the Traditional scheme. 


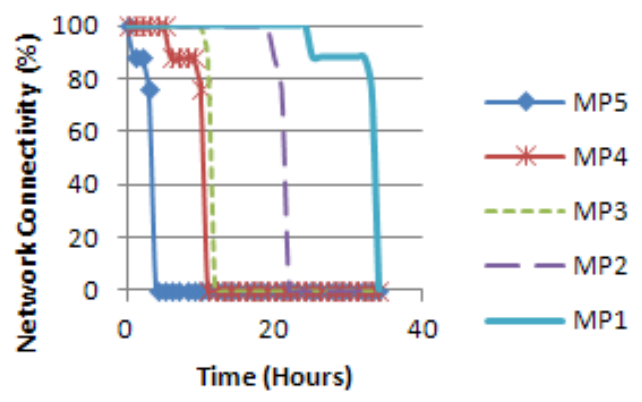

Fig. 7. Connectivity of the IDEALS|RMR scheme.

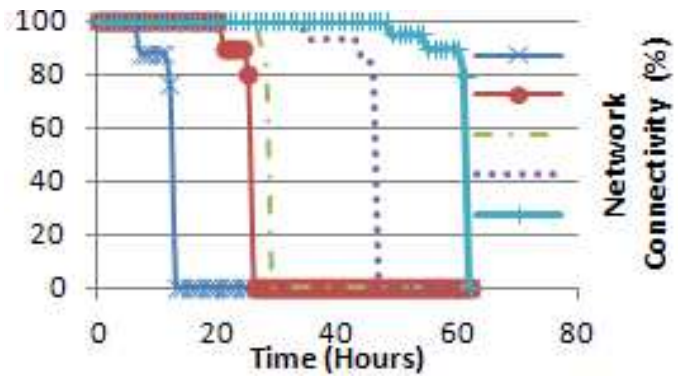

Fig. 8. Connectivity of the IRT scheme.

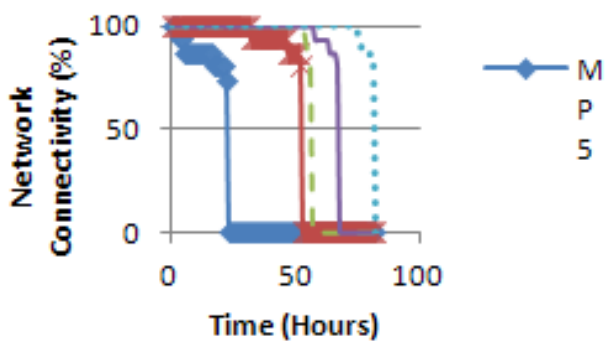

Fig. 9. Connectivity of the AIRT scheme.

(3) Message Loss (ML): It can be observed in fig. 5 that for all the schemes, the battery life drops drastically then it start dropping slowly, this is because of constant transmission of high priority messages. Consequently, low priority messages are ignored, leading to their loss.

As a summary, Table 3 shows the percentage improvement of the proposed AIRT scheme over traditional, IDEALS/RMR and IRT schemes in terms of network connectivity for the different types of message priorities. It is important to note that for the most important message (MP1), the AIRT scheme achieved $(82-9) / 9 * 100=811 \%$, $(82-34) / 34 * 100=141 \%$, and $(82-62) / 62=32 \%$ improvement over Traditional, IDEALS/RMR and IRT respectively. The same way is used to calculate the percentage improvement for the remaining message priorities.

Table 3. Percentage improvement of AIRT Scheme over all other Schemes in terms of Network Connectivity.

\begin{tabular}{llllll}
\hline & MP1 & MP2 & MP3 & MP4 & MP5 \\
\hline Traditional & 811 & 811 & 811 & 811 & 811 \\
IDEALS|PMR & 141 & 209 & 375 & 381 & 450 \\
IRT & 32 & 45 & 97 & 69 & 104 \\
\hline
\end{tabular}

\section{Conclusion}

In this paper, we introduced the AIRT scheme, which operates on the combination of information control (deciding if a message is worth processing based on the user defined rules, and how important the message is), energy management (balancing of the energy resource level and message importance) and TR adaptation (deciding on the transmission range based on nodes energy resource level and message importance) which we believe have never been considered before. Though scalability is not the primary concern of the work, but it is suitable for harsh environments. Simulation was performed using $\mathrm{C}$ programming language where a single node was simulated to show the operation of our scheme. The proposed scheme is preferred over others in situations when an intelligent system is used to adjust the TR of nodes. The results obtained show that the proposed AIRT scheme outperforms the other schemes (IRT, IDEALS|RMR, and Traditional) in terms of lifetime and connectivity, however, considerable amount of messages are lost due to TR adaptation.

We are currently working on coupling AIRT scheme with fuzzy logic, in the sense that, decisions on the MP, PP and TR values can be based on fuzzy logic, with the anticipation that message loss will be decreased.

\section{References}

[1] Akyildiz, I.F., Su, W., Sankarasubramaniam, Y., Cayirci, E.: Wireless sensor networks: a survey, Computer Networks. 38, 393-422 (2002).

[2] Raquel, A.F.M, Antonio, A.F.L, Badri, N.: The distinctive design characteristic of a wireless sensor network: the energy map. Computer Communications 27, 935-945 (2004).

[3] Anastasi, G., Conti, M., Francesco, M.D., Passarella, A.: Energy conservation in wireless sensor networks: A survey, Ad Hoc Networks. 7, 537-568 (2009).

[4] Jason, L. H., David, E.C.: MICA: a wireless platform for deeply embedded networks. IEEE Micro. 22, 12-24 (2002).

[5] Adel Gaafar, A.E., Hussein, A.E., Salwa, E.R., Magdy, M.I.: An Energy Aware WSN Geographic Routing Protocol, Universal Journal of Computer Science and Engineering Technology 1 (2), 105-111 (2010).

[6] Lo, B.P.L., Yang G-Z., Key technical challenges and current implementations of body sensor networks, in: Proceedings of the Second International Workshop Wearable and implantable Body Sensor Networks (BSN'2005), London, UK, April 2005.

[7] Werner-Allen, G., Lorincz, K., Ruiz, M. et al.: Deploying a wireless sensor network on an active volcano, Internet Computing, IEEE 10 18-25 (2006).

[8] Y. Liu, W.K.G. Seah, A priority-based multi-path routing protocol for sensor networks, in: Proceedings of the 15th IEEE international Symposium on Personal, Indoor and mobile Radio Communications (PIMPC'04), September 2004, pp.216-220. 
[9] Merrett, G.V., B.M. A`1-Hashimi, N.M., White, N.R. Harris, Information managed wireless sensor networks with energy aware nodes, in: Proceedings of the 2005 NSTI Nanotechnology Conference and Trade Show (Nano Tech'05), Anaheim, CA, 2005, pp. 367-370.

[10] Merrett, G.V., A`l-Hashimi, N.M., White, N.R.: Energy managed reporting for wireless sensor networks, Sensors and Actuators A 142, 379-389 (2008).

[11] Mingming, L., Jie, W., Mihaela, C., Minglu, L., EnergyEfficient Connected Coverage of Discrete Targets in Wireless Sensor Networks, International Journal of Ad Hoc and Ubiquitous Computing4, 137 - 147 (2009).
[12] Song, C., Liu, M., Cao, J., Zheng, Y., Gong, H., Chen, G.: Maximizing network lifetime based on transmission range adjustment in wireless sensor networks, Computer Communications 32, 1316-1325 (2009).

[13] Busse, M., Haenselmann, T., Effelsberg, W.: Energyefficient forwarding in wireless sensor Networks, Pervasive and Mobile Computing 4, 3-32 (2008).

[14] Abdullahi, A.I., Muhammed, S.: Energy-Aware Transmission Scheme for Wireless Sensor Networks: The Third International Conference on Wireless \& Mobile Networks. Ankara, Turkey, 2011. http://www.purplemath.com/modules/distform.htm. 